\title{
DESEMPENHO OPERACIONAL DE TRATOR COM E SEM O ACIONAMENTO DA TRAÇÃO DIANTEIRA AUXILIAR ${ }^{1}$
}

\author{
KIYOSHI YANAI ${ }^{2}$, GASTÃO MORAES DA SILVEIRA ${ }^{3}$, KLEBER PEREIRA LANÇAS ${ }^{4}$, ILA MARIA CORRÊA ${ }^{2}$ \\ e JOSÉ VALDEMAR GONZALEZ MAZIERO²
}

\begin{abstract}
RESUMO - Entre os fatores que podem influenciar o desempenho de tração do trator agrícola, destacam-se a pressão de inflação e a carga sobre o rodado motriz. Neste trabalho, o objetivo foi avaliar, em situação de campo, a influência dos fatores inflação (sob quatro níveis) e carga sobre o rodado motriz (sob quatro níveis), combinados com e sem o uso da tração dianteira auxiliar. O experimento, totalizando 32 tratamentos com três repetições, foi distribuído em blocos ao acaso. O trator estudado foi submetido a uma força de tração constante na barra de tração, imposta por outro trator. Os resultados evidenciaram a influência significativa da lastragem nos parâmetros patinagem e coeficiente de tração, que aumentaram com a redução da carga sobre o rodado. A pressão de inflação influiu significativamente nos parâmetros patinagem, velocidade de deslocamento e potência na barra, sem no entanto apresentar tendência de comportamento. $\mathrm{O}$ uso da tração dianteira auxiliar mostrou vantagens significativas em relação aos mesmos parâmetros anteriores. A interação dos fatores pressão de inflação e carga sobre o rodado mostrou que determinadas combinações de pressão foram mais favoráveis para o desenvolvimento de maior velocidade e menor patinagem. As características relacionadas ao desempenho do motor (consumo horário e rotação do motor) não foram afetadas por nenhum dos fatores e/ou suas interações.
\end{abstract}

Termos de indexação: lastragem, trator agrícola, barra de tração.

\author{
OPERATIONAL PERFORMANCE OF A TRACTOR \\ WITH AND WITHOUT THE USE OF WHEEL ASSIST DRIVE
}

\begin{abstract}
The factors that may have influence on tractor performance are several, inclusive tire load and inflation pressure. These factors were studied in field conditions (surface not disturbed), under four tire load, four inflation pressure with and without the use of the mechanical front wheel assist drive. A randomized complete block design with three replications was used. The tractor under evalution was submitted to a constant drawbar pull by other tractor linked with a load cell. The results showed the significant influence of load tire on the rear wheel slip and on coefficient of traction, which increased with the decreasing of the load tires. The inflation pressure influenced significantly the slip, the forward velocity and drawbar power without to show a general tendency in the behaviour. The use of mechanical front wheel assist drive showed significant advantages to the some prior parameters. The interactive effects of load tire and inflation pressure indicated that some combinations were more favourable to develop major velocity and minor slip. The characteristics concerning to engine performance (fuel consumption and speed engine) were not influenced by neither factors and/or their interactive.
\end{abstract}

Index terms: ballast, agricultural tractor, drawbar.

${ }^{1}$ Aceito para publicação em 9 de agosto de 1998.

Extraído da Dissertação de Mestrado do primeiro autor.

${ }^{2}$ Eng. Agr., M.Sc., Centro de Mecanização e Automação Agrícola/IAC/SAA, Caixa Postal 26, CEP 13201-970 Jundiaí, SP. E-mail: yanai@dea.iac.br

${ }^{3}$ Eng. Agr., Dr., IAC/SAA, Jundiaí, SP.

${ }^{4}$ Eng. Mec., Dr., Dep. de Eng. Rural, FCA/UNESP, Campus Lageado, Caixa Postal 237, CEP 18603-970 Botucatu, SP.

\section{INTRODUÇÃO}

A pressão de inflação e a carga sobre o rodado motriz, bem como o tipo de construção do rodado, a configuração da banda de rodagem, a superfície e condição do solo, são fatores que afetam o desempenho operacional do trator, e têm sido estudados isolados ou combinados, por diversos autores. 
Zambori (1967) estudou o efeito das cargas nos eixos e da pressão de inflação nos pneus em um trator de tração simples, sobre a força de tração na barra em solos arenosos. Para pressão de inflação do pneu com $78 \mathrm{kPa}$, foram aplicadas as cargas estáticas de $8,2,10,5,12,8,15,0$ e $17,9 \mathrm{kN}$ no eixo traseiro, e para carga estática de $7,06 \mathrm{kN}$, os pneus foram inflados com 19,6, 39,8, 58,8, 78,4 e 98,0 $\mathrm{kPa}$. O pesquisador concluiu que para tratores de tração simples, quando mantida a pressão constante, a lastragem beneficiou a força de tração, porém, o aumento da pressão de inflação mantida a carga constante, provocou um decréscimo na força de tração.

Burt et al. (1979) estudaram o efeito conjugado da carga dinâmica e da patinagem de dois tipos de pneus, um de 12.4-28 com quatro lonas, e outro, de 12.4-38 com seis lonas, com pressão de inflação constante de $110 \mathrm{kPa}$, em várias condições do solo. Três tipos de testes foram conduzidos para definir as curvas de desempenho do pneu, a saber: teste de tração zero variando a carga dinâmica; teste de carga dinâmica constante variando a velocidade de deslocamento e teste de velocidade de deslocamento constante variando a carga dinâmica. Os pesquisadores concluíram que, para uma patinagem constante, a eficiência de tração aumentou com a carga dinâmica em solos compactados, ocorrendo o inverso em solos mobilizados.

Burt \& Bailey (1982) estudaram um pneu radial 28.8R-38 para determinar o desempenho de tração, sob diversos níveis de carga e pressão de inflação do pneu. Os solos utilizados foram barro-argiloso Davidson e barro-argiloso Congaree, em duas condições de superfície: uma, mobilizada, e outra, compactada. Os pesquisadores concluíram que a eficiência de tração pode ser melhorada pela seleção apropriada dos níveis de carga e pressão de inflação dos pneus.

McAllister (1983) estudou a resistência ao rolamento de vários tipos de tração para máquinas e equipamentos agrícolas, variando a carga e pressão de inflação dos pneus. Todos os pneus foram calibrados na pressão indicada pelos fabricantes. $\mathrm{O}$ autor concluiu que o coeficiente de resistência ao rolamento diminui com a redução da pressão de inflação, com a redução da carga e com o aumento do diâmetro e largura do pneu. Conclusão semelhante é a de Charles (1984), que estudou as curvas de desempenho de um pneu 18.4R-38 sob três condições de carga sobre o pneu e cinco níveis de pressão em solo firme e em solo mobilizado. Embora não seja uma das conclusões do autor, observa-se também que nas duas condições de solo desse trabalho, mantendo-se a pressão constante, houve aumento da força de tração com o aumento da carga sobre o pneu, e que mantendo-se constante a carga sobre o pneu, houve aumento da força de tração e da eficiência de tração, com o aumento da pressão de inflação.

Uma comparação direta entre os trabalhos citados fica dificultada pelas diferentes condições de realização dos testes de cada um. Ressalta-se, no entanto, que com menos ou mais evidência, as pesquisas mostram o efeito dos fatores carga e pressão de inflação no desempenho dos pneus. É reconhecido o efeito do aumento de carga sobre o pneu no aumento da força de tração desenvolvida, mas não se pode deixar de investigar até que ponto os níveis de carga afetam o aproveitamento da potência do motor (eficiência de tração), levando-se em conta também o tipo de superfície do solo.

Fontana et al. (1986), em operação de campo com escarificador, compararam o desempenho de tratores com e sem tração dianteira, com e sem o uso de lastro dianteiro. Verificaram que houve aumento da velocidade de deslocamento com o uso da tração auxiliar, proporcionando um aumento médio de 5,76\% na capacidade operacional. Observaram também, um aumento de 5,82\% em média, no consumo horário de combustível com o uso da TDA.

Schlosser \& Dallmeyer (1988) compararam o desempenho de um trator no campo, utilizando duas técnicas de aração, com e sem o uso da tração dianteira auxiliar. Observaram, com o uso da TDA, um aumento de $17 \%$ na capacidade operacional efetiva e diminuição da patinagem.

Yanai et al. (1988), com base em dados levantados em ensaios de tratores em concreto, analisaram o desempenho de cinco tratores agrícolas com tração dianteira auxiliar, quando comparado com sua versão de tração simples: concluíram que o uso da tração dianteira causou um aumento de 33,3\%, em média, na força de tração e de 13,9\% no consumo horário. 
Hilbert et al. (1992) avaliaram o desempenho de tratores com tração simples e tração dianteira auxiliar com a mesma potência, variando a velocidade de deslocamento em condições de campo. Os testes apresentaram diferenças significativas entre os tratores de tração simples e os de tração dianteira auxiliar em relação à resistência ao rolamento, ao passo que a velocidade de deslocamento não afetou o seu desempenho.

Diante do panorama de experiências citadas, a maioria realizada em condições de solo do exterior, surgiu o interesse em levantar informações que contribuíssem para maior conhecimento nessa área, relacionadas a uma condição de campo nacional.

O trabalho foi realizado com o objetivo de avaliar o desempenho operacional de um trator agrícola com tração dianteira auxiliar, levando-se em consideração fatores de pressão e de lastragem.

\section{MATERIAL E MÉTODOS}

O experimento foi realizado em área do Instituto Agronômico, em Jundiaí, localizado a $23^{\circ} 10^{\prime} 52^{\prime \prime}$ latitude oeste, com altitude de 700 metros.

A superfície do solo, em repouso há cinco anos, com vegetação controlada pelo uso de roçadoras, apresentava cobertura vegetal composta de plantas daninhas diversas. A textura do solo, até a profundidade de $15 \mathrm{~cm}$, apresentava $15 \%$ de argila, $35 \%$ de silte, $22 \%$ de areia fina e $28 \%$ de areia grossa, o que caracteriza o solo como solo franco.

Foram utilizados dois tratores: o primeiro, denominado "trator de tração", foi o Massey Ferguson modelo 292 $4 \times 2$ com TDA, com potência máxima de $71,0 \mathrm{kw}$ a $2.200 \mathrm{~min}^{-1}$ (valores reduzidos pela Associação Brasileira de Normas Técnicas, 1985), torque máximo de 363 N.m a $1.400 \mathrm{~min}^{-1}$, transmissão com 12 marchas à frente. O segundo trator denominado de "trator de carga", foi um Massey Ferguson modelo 295 4x2 com TDA.

Foi adotado um delineamento fatorial $4 \times 4 \times 2$, distribuído em blocos ao acaso, com três repetições, totalizando 32 tratamentos (96 parcelas), sendo quatro níveis de pressão de inflação, quatro situações de lastragem e duas condições de auxílio da tração dianteira auxiliar, isto é, com a tração ligada e desligada, impondo-se ao trator uma força constante na barra de tração (em torno de $12,2 \mathrm{kN}$, com coeficiente de variação de $2,6 \%$ ). Cada parcela constituiuse de uma área de $125 \mathrm{~m}^{2}(2,5 \times 50 \mathrm{~m})$ cujos $10 \mathrm{~m}$ iniciais e finais ficaram reservados para manobras e estabilização de cargas.
Os níveis de pressão de inflação utilizados foram: $\mathrm{P} 1=165 \mathrm{kPa}\left(24 \mathrm{lbf} / \mathrm{pol}^{2}\right)$ nos pneus dianteiros, e $193 \mathrm{kPa}$ $\left(28 \mathrm{lbf} / \mathrm{pol}^{2}\right)$ nos pneus traseiros; $\mathrm{P} 2=138 \mathrm{kPa}\left(20 \mathrm{lbf} / \mathrm{pol}^{2}\right)$ nos pneus dianteiros e $165 \mathrm{kPa}\left(24 \mathrm{lbf} / \mathrm{pol}^{2}\right)$ nos pneus traseiros; $\mathrm{P} 3=110 \mathrm{kPa}\left(16 \mathrm{lbf} / \mathrm{pol}^{2}\right)$ nos pneus dianteiros e $138 \mathrm{kPa}\left(20 \mathrm{lbf} / \mathrm{pol}^{2}\right)$ nos pneus traseiros; $\mathrm{P} 4=83 \mathrm{kPa}$ $\left(12 \mathrm{lbf} / \mathrm{pol}^{2}\right)$ nos pneus dianteiros e $110 \mathrm{kPa}\left(16 \mathrm{lbf} / \mathrm{pol}^{2}\right)$ nos pneus traseiros.

Os níveis de carga sobre o rodado foram: $\mathrm{L} 1=$ peso total do trator: $51,28 \mathrm{kN}(5.227 \mathrm{kgf}) ; 31,46 \mathrm{kN}(3.207 \mathrm{kgf})$ no eixo traseiro; $19,84 \mathrm{kN}$ (2.020 kgf) no eixo dianteiro; L2 = peso total do trator: $48,47 \mathrm{kN}(4.941 \mathrm{kgf}) ; 30,47 \mathrm{kN}$ (3.106 kgf) no eixo traseiro; $18,00 \mathrm{kN}(1.835 \mathrm{kgf})$ no eixo dianteiro; L3 = peso total do trator: 45,69 kN (4.657 kgf); $29,07 \mathrm{kN}$ (2.963 kgf) no eixo traseiro; 16,62 kN (1.694 kgf) no eixo dianteiro; $\mathrm{L} 4=$ peso total do trator: $43,01 \mathrm{kN}$ (4.384 kgf); $27,98 \mathrm{kN}$ (2.852 kgf) no eixo traseiro; $15,03 \mathrm{kN}$ (1.532 kgf) no eixo dianteiro.

Os níveis de tração adotados foram: TS = trator operando com a tração dianteira desligada; $\mathrm{TD}=$ trator operando com a tração dianteira ligada.

O desempenho operacional do trator foi avaliado em função dos parâmetros: patinagem das rodas traseiras, velocidade de deslocamento, potência na barra, coeficiente de tração, consumo horário e rotação do motor.

Para determinação dos parâmetros de desempenho foram utilizados os seguintes equipamentos: a) célula de carga ALFA, 10 t, ligada a indicador digital Logodata e a um registrador numérico: para indicação da força de tração; b) rodas dentadas (com 20 ressaltos) e sensores indutivos instalados nas rodas motrizes traseiras do trator: para indicação do número de giros percorridos pelo rodado; c) roda odométrica - roda de bicicleta aro 20 , com roda dentada com 20 ressaltos e sensor indutivo - instalada atrás da roda dianteira esquerda do trator: para determinação da velocidade de deslocamento, da potência na barra (produto da força de tração pela velocidade de deslocamento) e para a determinação da patinagem (giros da roda de tração associados aos giros da roda odométrica); d) painel com seis totalizadores digitais e um cronômetro ligados em conjunto: para indicação do número de giros das rodas motrizes, roda odométrica e do tempo de percurso; e) medidor volumétrico de combustível construído e descrito por Maziero et al. (1992) ligado ao sistema de alimentação do trator.

Durante o experimento, o trator deslocou-se em 4a marcha, operado com a aceleração máxima. O cálculo dos parâmetros avaliados foi realizado por meio das equações descritas abaixo:

a) Consumo horário de combustível

$\mathrm{CH}=\frac{(\mathrm{Lf}-\mathrm{Li}) \cdot 4,5}{\mathrm{t}} \cdot 3,6$

Pesq. agropec. bras., Brasília, v.34, n.8, p.1427-1434, ago. 1999 
b) Velocidade de deslocamento

$\mathrm{VD}=(\mathrm{NGRO} \cdot \mathrm{CRO}) \cdot 3,6 / \mathrm{t}$

c) Patinagem das rodas motrizes

$\mathrm{P}=\frac{(\mathrm{NGRT} \cdot \mathrm{CRT})-(\mathrm{NGRO} \cdot \mathrm{CRO})}{(\mathrm{NGRT} \cdot \mathrm{CRT})} \cdot 100$

d) Potência na barra

$$
\mathrm{PB}=\frac{\mathrm{FT} \cdot \mathrm{VD}}{270} \cdot 0,7355
$$

e) Coeficiente de tração

e.1) trator no modo de tração simples

$$
\mathrm{CT}=\frac{\mathrm{FT}}{\mathrm{PET}+\mathrm{FT} \cdot \mathrm{HB} / \mathrm{DEE}}
$$

e.2) trator no modo de tração dianteira ligada

$\mathrm{CT}=\mathrm{FT} / \mathrm{PT}$

onde:

$\mathrm{CH}=$ consumo horário de combustível, em L/h;

$\mathrm{Lf}=$ leitura final do nível de combustível;

$\mathrm{Li}=$ leitura inicial do nível de combustível;

$\mathrm{t}=$ tempo de consumo, em $\mathrm{s}$;

$4,5=$ volume de combustível correspondente à unidade de leitura do nível de combustível no tubo graduado, em mL;

3,6 $=$ constante de conversão de unidades;

$\mathrm{VD}=$ velocidade de deslocamento, em $\mathrm{km} / \mathrm{h}$;

$\mathrm{NGRO}=$ número de giros da roda odométrica;

$\mathrm{CRO}=$ circunferência de rolamento da roda odométrica, em m;

$\mathrm{P}=$ patinagem das rodas motrizes traseiras, em \%;

NGRT = número de giros das rodas traseiras;

$\mathrm{CRT}=$ circunferência de rolamento das rodas traseiras, em m;

$\mathrm{PB}=$ potência na barra, em $\mathrm{kW}$;

$\mathrm{FT}=$ força de tração, em kgf;

270 e $0,7355=$ constantes de conversão de unidades;

$\mathrm{CT}=$ coeficiente de tração;

$\mathrm{PET}=$ peso estático traseiro, em kgf;

$\mathrm{HB}=$ altura da barra, em mm;

DEE = distância entre eixos, em mm;

$\mathrm{PT}=$ peso total do trator, em $\mathrm{kgf}$.

Os resultados de cada parâmetro foram tratados em delineamento fatorial, utilizando-se o teste de Tukey a 5\% de probabilidade para comparação das médias dos fatores e de suas interações.

\section{RESULTADOS E DISCUSSÃO}

O resultado geral da análise de variância dos parâmetros de desempenho é apresentado na Tabela 1 , onde se observa que a lastragem, a pressão de inflação, o uso da tração dianteira e suas interações não acusaram diferenças significativas em nível de $5 \%$ de probabilidade, relativa aos parâmetros força de tração, rotação do motor e consumo horário. $\mathrm{O}$ coeficiente de tração mostrou significância apenas em função dos fatores lastragem e tração.

A força de tração, embora tenha sido fator condicionante do ensaio, foi considerada aqui como parâmetro resultante do desempenho, apenas para comprovar que o nível de força de tração imposto ao trator em todos os tratamentos, fora de fato homogêneo.

A rotação do motor e o consumo horário, não apresentando diferenças significativas, indicam que o desempenho do motor não foi afetado pelos diferentes tratamentos, pelo menos para esse nível de carga imposta à barra de tração. É de se supor que um nível de força de tração que exija maior demanda de torque do motor cause alguma diferença, quando se considera o uso, ou não, da tração dianteira auxiliar.

Tendo-se verificado que os parâmetros patinagem, velocidade de deslocamento e potência na barra apresentaram diferenças significativas devidas a um ou mais fatores e/ou suas interações, desenvolveu-se a análise individual dos fatores, pela aplicação do teste de Tukey a 5\% de probabilidade para aqueles parâmetros.

\section{Influência do fator lastragem}

O resultado da aplicação do teste de Tukey às médias dos parâmetros em função do fator lastragem é apresentado na Tabela 2.

Os parâmetros patinagem e coeficiente de tração mostraram-se bastante influenciados pela lastragem, verificando-se aumentos de $14,5 \% \mathrm{e}$ $15,0 \%$, respectivamente quanto aos dois parâmetros, quando a lastragem diminuiu de $\mathrm{L} 1=51,28 \mathrm{kN}$ a L4 $=43,01 \mathrm{kN}$.

Tais resultados não deixam de ser esperados, pois o uso de lastro, sob certos limites, tem sido uma 
TABELA 1. Resultado geral da análise de variância dos parâmetros de desempenho operacional ${ }^{1}$.

\begin{tabular}{lcccccccc}
\hline \multirow{2}{*}{ Fatores de variação } & \multirow{2}{*}{$\begin{array}{c}\text { Graus de } \\
\text { liberdade }\end{array}$} & \multicolumn{7}{c}{ Parâmetros de desempenho } \\
\cline { 3 - 9 } & & FT & RM & CH & P & VD & PB & CT \\
\hline Lastragem & 3 & $\mathrm{~ns}$ & $\mathrm{~ns}$ & $\mathrm{~ns}$ & $\mathrm{~s}$ & $\mathrm{~ns}$ & $\mathrm{~ns}$ & $\mathrm{~s}$ \\
Pressão & 3 & $\mathrm{~ns}$ & $\mathrm{~ns}$ & $\mathrm{~ns}$ & $\mathrm{~s}$ & $\mathrm{~s}$ & $\mathrm{~s}$ & $\mathrm{~ns}$ \\
Tração & 1 & $\mathrm{~ns}$ & $\mathrm{~ns}$ & $\mathrm{~ns}$ & $\mathrm{~s}$ & $\mathrm{~s}$ & $\mathrm{~s}$ & $\mathrm{~s}$ \\
Lastragem x Pressão & 9 & $\mathrm{~ns}$ & $\mathrm{~ns}$ & $\mathrm{~ns}$ & $\mathrm{~s}$ & $\mathrm{~s}$ & $\mathrm{~ns}$ & $\mathrm{~ns}$ \\
Lastragem x Tração & 3 & $\mathrm{~ns}$ & $\mathrm{~ns}$ & $\mathrm{~ns}$ & $\mathrm{~ns}$ & $\mathrm{~ns}$ & $\mathrm{~ns}$ & $\mathrm{~ns}$ \\
Pressão x Tração & 3 & $\mathrm{~ns}$ & $\mathrm{~ns}$ & $\mathrm{~ns}$ & $\mathrm{~ns}$ & $\mathrm{~ns}$ & $\mathrm{~ns}$ & $\mathrm{~ns}$ \\
Lastragem x Pressão x Tração & 9 & $\mathrm{~ns}$ & $\mathrm{~ns}$ & $\mathrm{~ns}$ & $\mathrm{~ns}$ & $\mathrm{~ns}$ & $\mathrm{~ns}$ & $\mathrm{~ns}$ \\
\hline CV (\%) & & 2,71 & 0,53 & 3,66 & 11,59 & 1,56 & 3,47 & 2,51 \\
\hline
\end{tabular}

${ }^{1}$ FT: força de tração; RM: rotação do motor; CH: consumo horário de combustível; P: patinagem das rodas traseiras; VD: velocidade de deslocamento; PB: potência na barra; CT: coeficiente de tração; ns: não-significativo; s: significativo a 5\% de probabilidade pelo teste de Tukey.

TABELA 2. Médias de patinagem e coeficiente de tração para o fator lastragem 1 .

\begin{tabular}{lcccc}
\hline Parâmetro & \multicolumn{4}{c}{ Lastragem } \\
\cline { 2 - 5 } & $\mathrm{L} 1$ & $\mathrm{~L} 2$ & $\mathrm{~L} 3$ & $\mathrm{~L} 4$ \\
& $(51,28 \mathrm{kN})$ & $(48,47 \mathrm{kN})$ & $(45,69 \mathrm{kN})$ & $(43,01 \mathrm{kN})$ \\
\hline Patinagem (\%) & $9,55 \mathrm{~b}$ & $10,18 \mathrm{ab}$ & $10,27 \mathrm{ab}$ & $10,93 \mathrm{a}$ \\
Coeficiente de tração & $0,299 \mathrm{~d}$ & $0,314 \mathrm{c}$ & $0,326 \mathrm{~b}$ & $0,344 \mathrm{a}$ \\
\hline
\end{tabular}

opção para reduzir a patinagem, uma vez que proporciona melhor aderência dos pneus ao solo, segundo Buckingham (1976), citado por Schlosser et al. (1992), e facilitam sua flutuação. No caso do coeficiente de tração (relação entre força de tração e peso dinâmico no rodado motriz), também era de se esperar tal tendência, pois nessa relação o numerador mantinha-se constante, enquanto o denominador decrescia de L1 $(51,28 \mathrm{kN})$ a L4 $(43,01 \mathrm{kN})$.

\section{Influência do fator pressão de inflação}

O resultado da aplicação do teste de Tukey às médias dos parâmetros em função do fator pressão é apresentado na Tabela 3. Optou-se, por simplicidade de apresentação, indicar a pressão de inflação referenciada apenas aos pneus traseiros. Os níveis de pressão corretos são as combinações de pressão no eixo dianteiro e traseiro.

Conforme se observa, as médias de patinagem, velocidade de deslocamento e potência na barra em pelo menos uma das pressões de inflação empregadas, diferem entre si de forma significativa, sem no entanto indicar uma tendência de aumento ou diminuição com a redução da pressão.

Burt \& Bailey (1982), que estudaram o efeito da pressão de inflação e da carga sobre pneus, não enfatizaram a tendência por um comportamento. Ao concluírem que o desempenho dos pneus pode ser maximizado pela seleção adequada de níveis de pressão e de carga, estão, certamente, considerando a influência da interação entre os dois fatores, também comprovada no presente trabalho.

\section{Influência do fator tração}

O resultado da aplicação do teste de Tukey às médias dos parâmetros em função do fator tração é apresentado na Tabela 4. Pelos resultados constantes nessa tabela, é fácil observar que o uso da tração dianteira auxiliar foi positivo em termos de patinagem, velocidade de deslocamento e potência na barra. O coeficiente de tração, entretanto, foi mais alto quando o trator operou com tração apenas no eixo traseiro, tendo aumentado $47,9 \%$ de uma condição para outra. 
Fontana et al. (1986) detectaram aumento médio de $8,8 \%$ na velocidade de deslocamento, uma redução média de $16,9 \%$ para $10,3 \%$ no índice de patinagem, e de 5,8\% na capacidade operacional efetiva quando a tração dianteira auxiliar foi acionada, em operações de escarificação do solo. Schlosser \& Dallmeyer (1988), em experimento de campo com arado gradeador, mostram índices de patinagem menores, bem como capacidade operacional efetiva (parâmetro dependente da velocidade de operação) maior, com a opção TDA ligada.

Entende-se que tais vantagens de patinagem e velocidade de deslocamento ocorra com a TDA ligada, porque nessa condição o trator divide o esforço tratório em seus dois eixos motrizes, facilitando seu deslocamento. Sendo a força constante e, havendo ganho na velocidade, a potência na barra conseqüentemente, é também maior.

Justificam-se, por sua vez, os menores valores de coeficiente de tração (índice que apresenta o apro-

TABELA 3. Médias de patinagem, velocidade de deslocamento e potência na barra para o fator pressão de inflação ${ }^{1}$.

\begin{tabular}{lcccc}
\hline Parâmetro & \multicolumn{4}{c}{ Pressão de inflação nos pneus traseiros } \\
\cline { 2 - 5 } & $\begin{array}{c}\mathrm{P} 1 \\
(193 \mathrm{kPa})\end{array}$ & $\begin{array}{c}\mathrm{P} 2 \\
165 \mathrm{kPa})\end{array}$ & $\begin{array}{c}\text { P3 } \\
(138 \mathrm{kPa})\end{array}$ & $\begin{array}{c}\mathrm{P} 4 \\
110 \mathrm{kPa})\end{array}$ \\
\hline Patinagem $(\%)$ & $11,28 \mathrm{a}$ & $10,47 \mathrm{a}$ & $8,33 \mathrm{~b}$ & $10,86 \mathrm{a}$ \\
Velocidade de & & & & \\
deslocamento $(\mathrm{km} / \mathrm{h})$ & $4,80 \mathrm{~b}$ & $4,84 \mathrm{~b}$ & $4,92 \mathrm{a}$ & $4,74 \mathrm{c}$ \\
Potência na barra $(\mathrm{kW})$ & $16,37 \mathrm{ab}$ & $16,38 \mathrm{ab}$ & $16,62 \mathrm{a}$ & $16,05 \mathrm{~b}$ \\
\hline
\end{tabular}

${ }^{1}$ Médias seguidas da mesma letra, na linha, não diferem entre si a $5 \%$ de probabilidade pelo teste de Tukey.

TABELA 4. Médias de patinagem, velocidade de deslocamento, potência na barra e coeficiente de tração ${ }^{1}$.

\begin{tabular}{lrc}
\hline \multirow{2}{*}{ Parâmetro } & \multicolumn{2}{c}{ Tração } \\
\cline { 2 - 3 } & TS & TD \\
\hline Patinagem (\%) & $12,02 \mathrm{a}$ & $8,44 \mathrm{~b}$ \\
Velocidade de deslocamento $(\mathrm{km} / \mathrm{h})$ & $4,73 \mathrm{~b}$ & $4,93 \mathrm{a}$ \\
Potência na barra $(\mathrm{kW})$ & $16,10 \mathrm{~b}$ & $16,61 \mathrm{a}$ \\
Coeficiente de tração & $0,383 \mathrm{a}$ & $0,259 \mathrm{~b}$ \\
\hline & \\
&
\end{tabular}

Pesq. agropec. bras., Brasília, v.34, n.8, p.1427-1434, ago. 1999 veitamento de peso no(s) eixo(s) motriz(es), ocorrido com o uso da tração auxiliar pelo fato de a força de tração, sendo constante, dividir valores de peso bem diferenciados: na tração simples, o peso dinâmico é o peso do eixo motriz acrescido da transferência de peso do eixo dianteiro; com a tração ligada, o peso dinâmico considerado é o peso total do trator, o que apresenta um denominador de valor superior, resultando num quociente menor.

Os resultados dessa tendência de comportamento encontrados quanto ao coeficiente de tração neste trabalho conferem com os de Souza (1986) e Yanai et al. (1988), obtidos em ensaios de tratores agrícolas em pista de concreto, em condições de máxima tração na barra.

\section{Influência da interação lastragem-pressão de inflação}

Os resultados da aplicação do teste de Tukey às médias dos parâmetros em função da interação lastragem-pressão de inflação são apresentados nas Tabelas 5 a 7. Na Tabela 5 , apenas referentes às condições P2 e P3, observa-se uma tendência de os valores de patinagem diminuírem com o aumento da lastragem. Entretanto, determinadas combinações, tais como L1P3 $(51,28 \mathrm{kN} ; 138 \mathrm{kPa})$ e L2P3 $(48,47 \mathrm{kN} ; 138 \mathrm{kPa})$, representam o melhor desempenho do trator, pois em termos de patinagem apresenta os menores valores. Observa-se, também, que em quase todos os níveis de lastragem, a pressão P3 $(138 \mathrm{kPa})$ apresentou melhores resultados, isto é, menor patinagem.

Na Tabela 6, também se observa, apenas, para as pressões P2 e P3 uma tendência de os valores de velocidade de deslocamento aumentarem com o aumento da lastragem. Da mesma forma que para a patinagem, a pressão $\mathrm{P} 3(138 \mathrm{kPa})$ foi quase sempre o melhor valor, em termos de velocidade, em todos os níveis de lastragem (embora sem apresentar diferenças significativas); já as combinações L1P3 $(51,28 \mathrm{kN} ; 138 \mathrm{kPa})$ e L1P2 $(51,28 \mathrm{kN} ; 165 \mathrm{kPa})$ representam as melhores opções para desenvolver velocidade.

Quanto ao parâmetro coeficiente de tração observa-se, na Tabela 7, que não foi influenciado pelos níveis de pressão, pois eles não apresentaram 
diferenças significativas entre os diferentes níveis de lastragem, embora tenha havido a tendência de P1 (193 kPa) dar valor maior que as outras pressões.

Os melhores índices de coeficiente de tração foram obtidos com a lastragem L4 $(43,01 \mathrm{kN})$, particularmente as combinações L4P1 (43,01 kN; $193 \mathrm{kPa})$ e L4P4 (43,01 kN; $110 \mathrm{kPa})$.

Analisando os níveis de pressão, observa-se que em todos os níveis houve tendência de aumentar o coeficiente de tração, embora com diferenças nãosignificativas, quando se diminuiu a lastragem. Isto se explica pelo mesmo fato já comentado anteriormente: sendo a força de tração constante, à medida que se diminuiu a carga (denominador na equação que define coeficiente de tração) o quociente resultante aumenta.

Como já observaram Burt \& Bailey (1982), adequadas combinações de pressão e carga possibilitam maximizar o desempenho dos pneus. No presente trabalho, é possível admitir o mesmo. No caso,

TABELA 5. Médias de patinagem, em \%, para a interação lastragem-pressã $0^{1}$.

\begin{tabular}{lcccc}
\hline \multirow{2}{*}{ Fator } & \multicolumn{4}{c}{ Pressão de inflação nos pneus traseiros } \\
\cline { 2 - 5 } & $\begin{array}{c}\mathrm{P} 1 \\
(193 \mathrm{kPa})\end{array}$ & $\begin{array}{c}\mathrm{P} 2 \\
(165 \mathrm{kPa})\end{array}$ & $\begin{array}{c}\mathrm{P} 3 \\
(138 \mathrm{kPa})\end{array}$ & $\begin{array}{c}\text { P4 } \\
(110 \mathrm{kPa})\end{array}$ \\
\hline L1 $(51,28 \mathrm{kN})$ & $12,01 \mathrm{aA}$ & $8,31 \mathrm{cB}$ & $7,46 \mathrm{bB}$ & $10,42 \mathrm{bcA}$ \\
L2 $(48,47 \mathrm{kN})$ & $12,01 \mathrm{aA}$ & $9,17 \mathrm{bcB}$ & $8,02 \mathrm{abB}$ & $11,51 \mathrm{abA}$ \\
L3 $(45,69 \mathrm{kN})$ & $9,84 \mathrm{bBC}$ & $10,34 \mathrm{bB}$ & $8,51 \mathrm{abC}$ & $12,40 \mathrm{aA}$ \\
L4 $(43,01 \mathrm{kN})$ & $11,25 \mathrm{abB}$ & $14,05 \mathrm{aA}$ & $9,35 \mathrm{aC}$ & $9,09 \mathrm{cC}$ \\
\hline
\end{tabular}

${ }^{1}$ Médias seguidas da mesma letra maiúscula, na linha, e minúscula, na coluna, não diferem entre si, a $5 \%$ de probabilidade, pelo teste de Tukey; DMS, $5 \%=1,81 \%$.

TABELA 6. Médias de velocidade de deslocamento, $\mathrm{em} \mathbf{k m} / \mathbf{h}$, para a interação lastragem-pressão 1 .

\begin{tabular}{lllll}
\hline \multirow{2}{*}{ Fator } & \multicolumn{4}{c}{ Pressão de inflação nos pneus traseiros } \\
\cline { 2 - 5 } & \multicolumn{1}{c}{$\begin{array}{c}\mathrm{P} 1 \\
(193 \mathrm{kPa})\end{array}$} & $\begin{array}{c}\mathrm{P} 2 \\
(165 \mathrm{kPa})\end{array}$ & $\begin{array}{c}\mathrm{P} 3 \\
(138 \mathrm{kPa})\end{array}$ & $\begin{array}{c}\mathrm{P} 4 \\
(110 \mathrm{kPa})\end{array}$ \\
\hline L1 $(51,28 \mathrm{kN})$ & $4,72 \mathrm{cB}$ & $4,96 \mathrm{aA}$ & $4,97 \mathrm{aA}$ & $4,75 \mathrm{abB}$ \\
L2 $(48,47 \mathrm{kN})$ & $4,77 \mathrm{bcBC}$ & $4,88 \mathrm{abAB}$ & $4,94 \mathrm{aA}$ & $4,70 \mathrm{bC}$ \\
L3 $(45,69 \mathrm{kN})$ & $4,89 \mathrm{aA}$ & $4,82 \mathrm{bcA}$ & $4,92 \mathrm{aA}$ & $4,66 \mathrm{bB}$ \\
L4 $(43,01 \mathrm{kN})$ & $4,84 \mathrm{abA}$ & $4,72 \mathrm{cB}$ & $4,86 \mathrm{aA}$ & $4,87 \mathrm{aA}$ \\
\hline
\end{tabular}

${ }^{1}$ Médias seguidas de mesma letra maiúscula, na linha, e minúscula, na coluna, não diferem entre si, a $5 \%$ de probabilidade, pelo teste de Tukey; DMS, $5 \%=0,12 \mathrm{~km} / \mathrm{h}$
TABELA 7. Médias de coeficiente de tração para a interação lastragem-pressão ${ }^{1}$.

\begin{tabular}{lcccc}
\hline \multirow{2}{*}{ Fator } & \multicolumn{4}{c}{ Pressão de inflação nos pneus traseiros } \\
\cline { 2 - 5 } & $\begin{array}{c}\text { P1 } \\
(193 \mathrm{kPa})\end{array}$ & $\begin{array}{c}\text { P2 } \\
(165 \mathrm{kPa})\end{array}$ & $\begin{array}{c}\text { P3 } \\
(138 \mathrm{kPa})\end{array}$ & $\begin{array}{c}\text { P4 } \\
(110 \mathrm{kPa})\end{array}$ \\
\hline L1 $(51,28 \mathrm{kN})$ & $0,303 \mathrm{cA}$ & $0,297 \mathrm{dA}$ & $0,301 \mathrm{cA}$ & $0,297 \mathrm{dA}$ \\
L2 $(48,47 \mathrm{kN})$ & $0,317 \mathrm{bA}$ & $0,313 \mathrm{cA}$ & $0,313 \mathrm{bA}$ & $0,313 \mathrm{cA}$ \\
L3 $(45,69 \mathrm{kN})$ & $0,323 \mathrm{bA}$ & $0,327 \mathrm{bA}$ & $0,328 \mathrm{aA}$ & $0,329 \mathrm{bA}$ \\
L4 $(43,01 \mathrm{kN})$ & $0,348 \mathrm{aA}$ & $0,344 \mathrm{aA}$ & $0,340 \mathrm{aA}$ & $0,345 \mathrm{aA}$ \\
\hline
\end{tabular}

1 Médias seguidas da mesma letra maiúscula, na linha, e minúscula, na coluna, não diferem entre si, a $5 \%$ de probabilidade, pelo teste de Tukey; DMS, $5 \%=0,012$.

as combinações L1P3 (L1 = 51,28 kN; $\mathrm{P} 3=138 \mathrm{kPa}$ ) permitem que o trator opere desenvolvendo maior velocidade de deslocamento com menor patinagem das rodas motrizes, porém com algum prejuízo, na eficiência de conversão de peso, se considerarmos que nesse nível de pressão o coeficiente de tração diferiu estatisticamente dos outros níveis de lastragem.

\section{CONCLUSÕES}

1. Mantendo-se uma força de tração constante de $12,2 \mathrm{kN}$, os níveis de lastragem e os níveis de combinação de pressão de inflação estudados, com e sem o uso da tração dianteira auxiliar, não afetam a rotação do motor e o consumo horário de combustível do trator MF 292 aux.

2. O aumento dos níveis de lastragem reduz a patinagem e o coeficiente de tração.

3. As combinações de pressão de inflação influenciam significativamente a patinagem, a velocidade de deslocamento e a potência na barra, não mostrando, porém, tendência de aumento ou redução com seu decréscimo.

4. O uso da tração dianteira auxiliar influenciam significativa e positivamente a patinagem, a velocidade de deslocamento e a potência na barra, sendo a tração simples mais eficiente apenas para o desenvolvimento de maior coeficiente de tração.

5. Determinadas combinações de pressão e lastragem proporcionam melhores valores de patinagem, velocidade de deslocamento e coeficiente de tração. 


\section{REFERÊNCIAS}

ASSOCIAÇÃO BRASILEIRA DE NORMAS TÉCNICAS. Motores alternativos de combustão interna de ignição por compressão (diesel) ou ignição por centelha (otto) de velocidade angular variável. Ensaio. Método de ensaio NBR 5484. Rio de Janeiro, 1985. 8p.

BURT, E.C.; BAILEY, A.C. Load and inflation pressure effects on tires. Transactions of the ASAE, St. Joseph, v.25, n.4, p.881-884, 1982.

BURT, E.C.; BAILEY, A.C.; PATTERSON, R.M.; TAYLOR, J.H. Combined effects of dynamic load and travel reduction on tire performance. Transactions of the ASAE, St. Joseph, v.22, n.1, p.40-45, 1979 .

CHARLES, S.M. Effects of ballast and inflation pressure on tractor tire performance. Agricultural Engineering, v.65, n.2, p.11-13, 1984.

FONTANA, C.F.; DALLMEYER, A.U.; POZZERA, J.; WEISS, A. Desempenho comparativo de tratores com e sem tração dianteira auxiliar durante a escarificação do solo. Revista do Centro de Ciências Rurais, Santa Maria, RS, v.16, n.3, p.237-249, 1986.

HILBERT, J.; BALBUENA, R.G.; CLAVERIE, J. Medición y predicción de la resistencia a la rodadura en tractores de tracción simple y tracción delantera assistida en laboreo secundario. In: CONGRESSO BRASILEIRO DE ENGENHARIA AGRÍCOLA, 21., 1992, Santa Maria, RS. Anais... Santa Maria: UFSM/SBEA, 1992. v.3, p.1742-1752.

McALLISTER, M. Reduction in the rolling resistance of tires for trailed agricultural machinery. Journal of
Agricultural Engineering Research, Silsoe, v.28, p.127-137, 1983.

MAZIERO, J.V.G.; CORRÊA, I.M.; YANAI, K.; MENEZES, J.F. de. Equipamento para medição do consumo de combustível em experimentos agrícolas. Bragantia, Campinas, v.51, n.2, p.197-202, 1992.

SCHLOSSER, J.F.; DALLMEYER, A.U. Desempenho operacional de um trator com tração dianteira auxiliar operando com as rodas dentro e fora de sulco. In: CONGRESSO BRASILEIRO DE ENGENHARIA AGRÍCOLA, 17.,1988, Iperó. Anais...Iperó: CENEA/SBEA, 1988. v.2, p.432-437.

SCHLOSSER, J.F.; PEREIRA, C.F.; SOUZA FILHO, E.G de; HURTADO, R.R.; MEZZOMO, C.P.L. Equalização da patinagem provocada pela diferenciação de lastros no trabalho do trator em aração a duas velocidades. In: CONGRESSO BRASILEIRO DE ENGENHARIA AGRÍCOLA, 21., 1992, Santa Maria, RS. Anais... Santa Maria: UFSM/SBEA, 1992. v.2, p.1535-1547.

SOUZA, E.G. Desempenho de tratores agrícolas. In: CONGRESSO BRASILEIRO DE ENGENHARIA AGRÍCOLA, 15., 1986, São Paulo. Anais... São Paulo: UNESP/SBEA, 1986. v.2, p.135-156.

YANAI, K.; CORRÊA, I.M.; MAZIERO, J.V.G.; MENEZES, J.F. de; PECHE, A.T.M. Desempenho comparativo de tratores com e sem tração dianteira auxiliar em pista de concreto. In: CONGRESSO BRASILEIRO DE ENGENHARIA AGRÍCOLA, 17., 1988. Iperó. Anais... Iperó: CENEA/SBEA, 1988. v.2, p.438-444.

ZAMBORI, J. Drawbar pull tests of various traction devices on sandy soils. Journal of Terramechanics, Pergamon, v.4, n.1, p.9-17, 1967. 